DOI: https://doi.org/10.46296/yc.v4i7.0039

\title{
MODELOS DE GESTIÓN DE CALIDAD PARA EL DESARROLLO DE EMPRENDIMIENTOS EN LAS INSTITUCIONES DE EDUCACIÓN SUPERIOR DE MANABÍ
}

\section{QUALITY MANAGEMENT MODELS FOR THE DEVELOPMENT OF ENTREPRENEURSHIP IN THE INSTITUTIONS OF HIGHER EDUCATION OF MANABÍ}

\author{
Silva-Gorozabel Jhon Jairo ${ }^{1 *}$; Feijó-Cuenca Nilba Priscila²; Feijó-Cuenca Tito Eliecer³ \\ ${ }^{1}$ Universidad Técnica de Manabí, UTM. Instituto de Posgrado. Portoviejo, Ecuador. \\ 2Universidad Técnica de Manabí, UTM. Portoviejo, Ecuador. \\ ${ }^{3}$ Universidad Técnica de Manabí, UTM. Portoviejo, Ecuador.
}

*Correo: jsilva5667@utm.edu.ec

\begin{abstract}
Resumen
El presente artículo tiene como objetivo analizar cinco modelos de gestión de la calidad para su actuación frente a la generación de emprendimientos de las Instituciones de Educación Superior en la provincia de Manabí. La metodología aplicada es de tipo descriptiva, constó de una revisión bibliográfica de cinco modelos de gestión de la calidad y fomento al emprendimiento que incluyen el cambio organizacional dentro de sus planes de estudio en diversas áreas. Los resultados permiten concluir que los modelos de gestión de la calidad son una guía para establecer una orientación coherente y estructurada del diagnóstico, la mejora continua y las acciones a seguir hacia la búsqueda de la excelencia, en el caso de Ecuador y específicamente en Manabí, los modelos adaptables son el modelo ISO 9000 y el modelo lberoamericano que abarcan principios y dimensiones de mejora continua, trabajo en equipo, participación, compromiso y desarrollo como clave de crecimiento empresarial.
\end{abstract}

Palabras clave: Formación, emprendimiento, innovación, instituciones de educación superior, modelos de gestión.

\begin{abstract}
The objective of this article is to analyze five quality management models for their performance against the generation of enterprises of Higher Education Institutions in the province of Manabí. The methodology applied is descriptive, it consisted of a bibliographic review of five models of quality management and promotion of entrepreneurship that include organizational change within their study plans in various areas. The results allow us to conclude that the quality management models are a guide to establish a coherent and structured orientation of the diagnosis, continuous improvement and the actions to follow towards the search for excellence, in the case of Ecuador and specifically in Manabí. The adaptable models are the ISO 9000 model and the IberoAmerican model that encompass principles and dimensions of continuous improvement, teamwork, participation, commitment, and development as a key to business growth.
\end{abstract}

Keywords: Training, entrepreneurship, innovation, higher education institutions, management model.

Información del manuscrito:

Fecha de recepción: 05 de mayo de 2020

Fecha de aceptación: 08 de julio de 2020

Fecha de publicación: 10 de julio de 2020 


\section{Introducción}

El contexto económico actual impone a los países en vías de desarrollo a generar la promoción continua $y$ fortalecimiento del emprendimiento, ya que es un tema con grandes beneficios, pero también de grandes compromisos desde el sector gubernamental, social, empresarial y académico, toda vez que su impulso incide en el incremento del empleo y en el crecimiento económico. Desde una perspectiva académica situada en la educación superior Centeno (2017), destaca: "muchos países están adoptando políticas amigables de emprendimiento, no obstante, éstas aún son insuficientes porque no cuentan con un sistema regulatorio que fomente la innovación, incentivos fiscales, acceso a financiamiento 0 a un sistema académico que direccione eficazmente al emprendedor" (p.18).

Zamora (2017) y Maldonado (2019), con un enfoque situado en la realidad de nuestro país, afirman que, en Ecuador los emprendimientos se incrementan con indicadores mayores al nivel de América Latina (p.16), contando además con una normativa legal de impulso a la actividad empresarial donde la academia y el sector público se mantienen en movimiento (p.12).

Bajo esta premisa es claro comprender que el gran reto de las instituciones de educación superior es mejorar la calidad de los emprendimientos, buscando que no estén marcados por la necesidad sino por la oportunidad, ya que al surgir de la necesidad resta calidad en las ideas y potencial de crecer no solo en el país sino fuera de él, para esto, las ideas deben contener un alto valor añadido que generen importe para los consumidores.

Ante este panorama se evidencia la necesidad de construir alianzas proactivas en las que interactúen el mundo empresarial, el académico y el estado; por ello, las Instituciones de Educación Superior (IES) no deben ser solo productoras de conocimiento aisladas del contexto social, sino que deben articularse con planes, programas y proyectos que impacten positivamente en el desarrollo socioeconómico, ya que en la medida en que sus procesos académicos sean pertinentes, contribuirán a solucionar los problemas de la región, además de 
Revista Científica Multidisciplinaria Arbitrada YACHASUN. Volumen 4, Número 7 (jul-dic) ISSN: 2697-3456 Modelos de gestión de calidad para el desarrollo de emprendimientos en las instituciones de educación superior de Manabi.

responder al avance de la ciencia con actitud emprendedora.

Desde luego, las IES de la provincia de Manabí, se convierten en polos de desarrollo que contribuyen al fortalecimiento de la región mediante sus procesos de investigación, emprendimiento e innovación, comprometidas con el progreso social, cultural, ambiental y económico del país. Por ello, es necesaria la identificación de modelos de gestión de calidad afines a la realidad circundante, que potencien el emprendimiento en los estudiantes hacia iniciativas empresariales de alto impacto.

Las brechas existentes entre el sector educativo superior y el sector empresarial parten del desequilibrio existente debido al alto nivel de desempleo y la baja demanda laboral; brecha que se vería significativamente disminuida con la creación de empresas que propicien oportunidades de crecimiento económico y mejoren la estabilidad de las familias.

La innovación desde las instituciones de educación superior promueve la cultura emprendedora, fortaleciendo la creatividad e inspiración particular de una idea para solucionar un problema o una situación que lo demande a causa de una necesidad (Jaime Carriel, 2017, p.7). Esta visión holística aplicada en la zona 4 (provincias de Manabí y Santo Domingo) promovería la articulación de los espacios de innovación y transferencia de tecnología existentes, fortaleciendo el trabajo en red entre las IES que, de acuerdo con la Secretaría de Educación Superior, Ciencia, Tecnología e Innovación (SENESCYT), son descritos como espacios colaborativos que fomentan la innovación, el emprendimiento y la transferencia de conocimiento, aportando al desarrollo productivo del país y potencializando la economía con un efecto diferenciador en la sociedad y sirviendo de enlace en la construcción de conocimiento desde su formación práctica y aportando en los procesos de I+D en las empresas vinculadas.

\subsection{Antecedentes}

La procedencia del emprendimiento como recurso generador de riquezas tiene su origen significativo, según, Guananga I., (2018), explica: EI término emprender viene del latín "IN" que representa iniciar, también 
emprendimiento del viene del francés "entrepreneur" que significa pionero; estos términos permiten asociar la idea de emprender con iniciar, crear, innovar, es decir, que el emprendimiento incluye actividades de creación, innovación e iniciación donde surgen otras preguntas como: qué, para qué y cómo emprender ( $p$. 9). Por su parte, Lasio, et. al., (2017), indican que; "la importancia del emprendimiento surgió como un resultado para la administración pública, empresas privadas y la academia" (p. 9).

Desde la perspectiva de los autores, se concuerda que constituye un proceso de transformación que compromete a los entes universitarios que ha pasado a llamarse Universidad Emprendedora, es decir, que la universidad se debe convertir en un potencial para contribuir, al desarrollo económico y social a través de los espacios de investigación, de medición del nivel de actividad y de los factores que identifican las áreas de oportunidad para permitir la creación de políticas públicas y recomendaciones para mejorar el nivel de actividad emprendedora.

\subsection{Definición de los modelos gestión de calidad}

Autores como, Camison, Cruz y Gonzalez (2006) definen a los modelos de Gestión de la Calidad como "marcos que brindan consejo y guía sobre como operativizar y poner en práctica los principios, las prácticas y los métodos de control, gestión y mejora de la calidad, desde un cierto enfoque" (p.36). Por otra parte, Giorgetti, Romero, y Vera (2014) manifiestan que "los modelos de gestión de la calidad se proponen medir el nivel de eficiencia de las instituciones educativas" (p.5).

En resumen, un modelo de gestión de la calidad es un instrumento que sirve para medir los indicadores de eficiencia y eficacia que permitan someter los procesos a características distintivas del fenómeno y objeto de estudio, las cuales son idóneas de identificarse y medirse en un plano operativo, a partir de las cuales se obtienen los datos definidos como necesarios para la evaluación, incluyendo también las variables para la operativización, evaluación, construcción y elaboración de los instrumentos de medición. 


\subsection{Modelos de gestión de calidad adaptados al emprendimiento}

Según manifiesta González (2014), "los Modelos de Gestión de los emprendimientos son una forma de organizar, combinar los recursos, cumplir con los objetivos, principios, políticas, sistemas, procesos, procedimientos y pautas de comportamiento para conseguir los resultados esperados y mejorar el desempeño de la organización" ( $p$. 43).

En consecuencia, estos elementos se encuentran en las normas internacionales de la gestión de la calidad que enmarcan los objetivos misionales, los procesos básicos de operación, la estructura y organización, la cultura organizacional, las políticas y competencias del talento humano y la planeación estratégica formal de la organización, tales como las Normas ISO 9000 y los modelos de Excelencia, que permiten establecer un sistema de gestión basado en la calidad y calidad total.

\subsubsection{Modelo ISO 9000}

Según indica Sáinz (2018), "en 1987 se publican las Normas ISO
9000, que son conjuntos de cánones de carácter voluntario sobre el aseguramiento de la calidad de procesos, y se consolidan a nivel internacional el marco normativo de gestión y control de la calidad" ( $p$. 46).

La Norma ISO 9001:2015, hace referencia al Sistema de Gestión de la Calidad (SGC) en una organización e indica que se deben implantar de acuerdo a los siguientes principios:

- Enfoque al cliente: describe el enfoque de cumplir con los requisitos del cliente y tratar de exceder sus expectativas.

- Liderazgo: enfatiza el liderazgo en todos los niveles que establecen la unidad de propósito y la dirección.

- Compromiso de las personas: menciona el empoderamiento $y$ compromiso en toda la organización para incrementar la capacidad de generar y proporcionar valor.

- Enfoque a procesos: entiende y gestiona los procesos interrelacionados que funcionan como un sistema coherente.

- Mejora: se basa en un enfoque continuo hacia la mejora. 
- Toma de decisiones basada en la evidencia: las decisiones basadas en el análisis y la evaluación de datos e información tienen mayor probabilidad de producir los resultados deseados.

- Gestión de las relaciones: mide el éxito sostenido con base a las relaciones con las partes interesadas pertinentes, tales como los proveedores.

En resumen, se conoce que el modelo ISO 9000 y la norma ISO 9001:2015 es la base o punto de partida para comprender las normas y definir los términos fundamentales utilizados que se necesitan para evitar malentendidos en su utilización.

\subsubsection{El modelo del Premio Deming}

Según Castillo (2019), el ciclo de Deming consiste en 4 etapas: planificar, hacer, verificar y actuar (PHVA) por medio del cual se identifica el problema, se determinan sus posibles soluciones, se comprueba la eficacia de estas y se generaliza el sistema que se puede emplear en procesos y proyectos de las organizaciones.
En este sentido, el modelo permitirá a las IES lograr la satisfacción de los estudiantes, vistos como clientes internos, la mejora continua de los procesos del servicio educativo relacionado con el emprendimiento, lo que propicia la satisfacción del cliente garantizando la evolución y mejora continua institucional.

\subsubsection{El modelo de Premio Baldrige}

Rodríguez y Sandoval (2017), describen que "el modelo Malcolm Baldrige hace referencia a que las organizaciones demuestren una gestión de la calidad efectiva, mediante la formación y la implicación de los empleados, en la mejora de la empresa (p. 3). Este modelo está compuesto por el perfil organizacional y por siete criterios:

1. Liderazgo: donde se destaca la participación en la creación de estrategias, de sistemas y de métodos para el logro de la excelencia.

2. Planeamiento estratégico: para desplegar los objetivos de estándares de calidad, eficiencia y competitividad con enfoque definido $y$ planeamiento estratégico y de mejora continua. 
3. Orientación hacia el cliente y el mercado: examina los requerimientos, necesidades, expectativas y las preferencias de los clientes y de los mercados.

4. Medición, análisis y gestión del conocimiento: inspecciona el desempeño de la organización frente a la sociedad en base a datos confiables.

5. Enfoques a los recursos humanos: examina la forma en que los empleados utilizan su potencial en alineación con los objetivos organizacionales, la estrategia y los planes de acción.

6. Gestión de procesos: examina los aspectos claves del proceso de gestión de la organización para crear valor a los consumidores y a la entidad.

7. Resultados: mide el desempeño y la mejora en pro de la satisfacción del cliente, del mercado, la orientación hacia las personas y la responsabilidad social.

En síntesis, el modelo establece que los líderes de una organización corresponden a estar orientados a la dirección estratégica y a los clientes en una mejora en las relaciones de los empleados, dando mayor productividad y satisfacción de los clientes, que permite proporcionar mayor valor a los clientes y grupos de interés, contribuyendo a la sostenibilidad, la eficacia y capacidades de toda la organización y su personal.

\subsubsection{El modelo EFQM (Modelo} Europeo de Excelencia Empresarial) de Excelencia

El modelo EFQM está integrado por 3 elementos: valores de excelencia, criterios y el esquema lógico REDER, que interactúan entre sí y ha permitido entender la causa y efecto entre lo que hace una organización y los resultados que consigue (Chacon, C. y Rugel, 2018, p.4).

Este modelo constituye una herramienta de gestión que permite evaluar el rendimiento de la organización y esquema dinámico que permite conducir sistemáticamente las mejoras de todas las áreas de una organización, considerando criterios de excelencia referente al: liderazgo, estrategias, personas, alianzas y recursos, 
procesos, productos-servicios y resultados, que permite examinar a la organización en su conjunto para adoptar una perspectiva holística a fin de alcanzar el éxito, reforzar su liderazgo y establecer estrategias de mejora.

\subsubsection{Modelo Iberoamericano de Excelencia en la Gestión}

Serna (2019), explica que "el modelo facilita la comprensión de los principios y la contextualización de las prácticas de la gestión de la calidad total TQM en empresas" (p.383).

En síntesis, el Modelo Europeo de Excelencia Empresarial EFQM tiene como objetivo identificar los comportamientos que se deben tener en cuenta para el análisis de gestión, a partir de un instrumento de recolección de información que incluye 6 dimensiones que se denominan: gestión estratégica, gestión táctica, gestión de empleados, gestión de clientes, gestión de proveedores y gestión de recursos a fin de promover $y$ desarrollar la gestión de la calidad en los e integrar experiencia y conocimiento de otros países hacia las entidades en relación al desarrollo de modelos y sistemas de excelencia que otorguen beneficios de sus miembros en relación a la mejora de competitividad y posición internacional a través de la plena satisfacción de sus clientes internos y externos.

\section{Metodología (Materiales y métodos)}

El presente trabajo de investigación, pertenece a una tipología descriptiva de característica cualitativa, basada en la revisión bibliográfica y analítica, con el objetivo de caracterizar sustentos teóricos sobre los modelos de gestión de calidad para el desarrollo de emprendimientos en las Instituciones de Educación Superior de Manabí, a fin de elevar el nivel de competitividad institucional y vincular a la academia con el sector empresarial mediante la gestión de calidad de los emprendimientos que en ellas se generen.

Constó de una revisión bibliográfica de cinco modelos de gestión de la calidad y fomento al emprendimiento utilizados en algunas IES entre los años 2010-2020 que incluyen el emprendimiento dentro de sus planes de estudio en diversas áreas. De este modo, se utilizaron fuentes de información secundarias tales 
Revista Científica Multidisciplinaria Arbitrada YACHASUN. Volumen 4, Número 7 (jul-dic) ISSN: 2697-3456 Modelos de gestión de calidad para el desarrollo de emprendimientos en las instituciones de educación superior de Manabi.

como: artículos, revistas el acceso a nuevos mercados a nivel electrónicas, normativas internacionales, libros, tesis 0 trabajos de posgrado, con el propósito de comprender el enfoque de los modelos de gestión adaptados a los emprendimientos por parte de las IES que contribuyan y sustenten procesos de mejora.

\section{Resultados}

El ámbito de gestión de los emprendimientos en las instituciones de educación superior concentran su atención en actividades como la promoción de los vínculos, apoyo científico y tecnológico a las empresas y microempresas, promoción I+D, creación de empresas con base tecnológica, e incubadoras y formación del talento humano, en este sentido, requieren direccionar su plan formativo hacia la innovación y desarrollo sostenible promoviendo la gestión de la calidad a partir de modelos significativos que tributen a una visión holística de la universidad.

En el presente artículo se mencionan 5 modelos de gestión de la calidad, entre ellos: el modelo ISO 9000 que es una herramienta estratégica que permite a las IES gestionar la calidad, obteniendo como resultado 


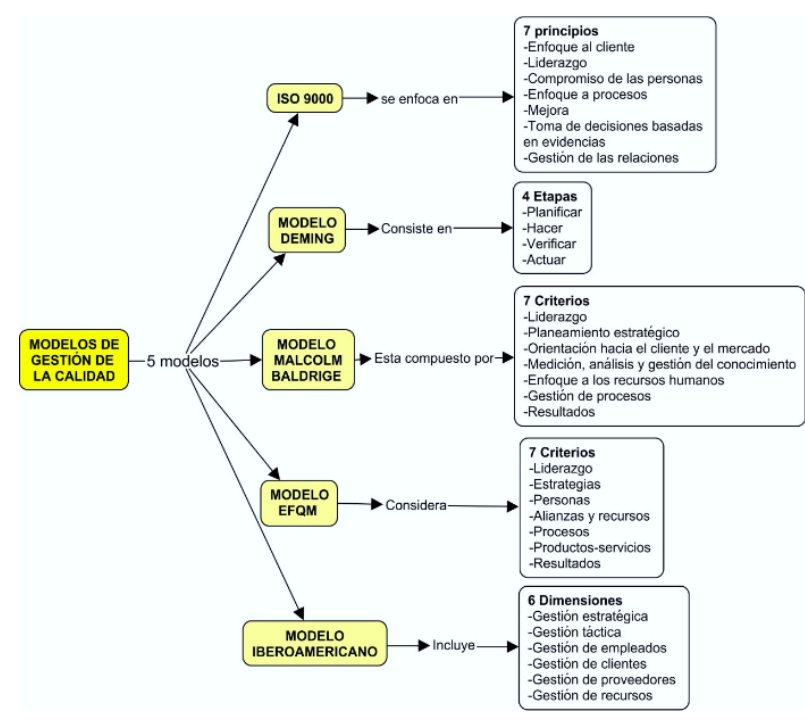

Fig. 1. Modelos de gestión de la calidad en la Educación Superior.

Elaborado por: Autores

Los modelos de gestión de la calidad adaptados a las IES presentados, proporcionan enfoque y criterios básicos para analizar las características de los procesos y evaluación de la calidad de las instituciones de educación superior; sin embargo, su interpretación depende del modelo estratégico, los objetivos institucionales y la metodología empleada para no sólo alcanzarla, sino para producirla.

\section{Conclusiones}

Los modelos de gestión de la calidad son una guía para establecer una orientación coherente y estructurada del diagnóstico, la mejora continua y las acciones a seguir hacia la búsqueda de la calidad respecto a los bienes o servicios que ofrece la organización.

La gestión de los emprendimientos por parte de las instituciones de educación superior debe estar cimentada sobre alguno de los modelos de gestión de la calidad, en el caso de Ecuador, los modelos más usados son el modelo ISO 9000 y el modelo Iberoamericano que abarcan principios y dimensiones de la empresa en su búsqueda de la mejora continua, considerando como principales entradas a las personas e insumos que son fundamentales en la colaboración, el trabajo en equipo, la participación, el compromiso y el desarrollo como clave de crecimiento y enriquecimiento de la empresa y su gestión.

Los modelos de gestión analizados en el presente estudio ofrecen elementos necesarios para definir los principios de la calidad total, donde la satisfacción del cliente es el objetivo principal, y para esto se fundamentan en la mejora de los procesos, la adecuada toma de decisiones que conllevan a que el estudiante de nivel superior adquiera una visión holística de desarrollo para la gestión de emprendimientos 
de calidad sostenibles en el tiempo a fin de generar propuestas que den una solución efectiva a los problemas actuales del mercado y prevengan dificultades posteriores.

\section{Bibliografía}

Castillo, L. (2019). El modelo Deming (PHVA) como estrategia competitiva para realzar el potencial administrativo. Universidad Militar Nueva Granada.

http://repository.unimilitar.edu .co/handle/10654/34875

Centeno, A. (2017). El emprendimiento internacional en paises en desarrollo: Factores determinantes de la predicción del emprendimiento internacional. Madrid, España: Universidad Carlos III de Madrid. https://earchivo.uc3m.es/bitstream/ha ndle/10016/25418/Tesis_adol fo_centeno_2017.pdf?sequen $\mathrm{ce}=1$ \&isAllowed $=\mathrm{y}$

Chacon, C. y Rugel, K. (2018). Teorías, Modelos y Sistemas de Gestión de calidad. Espacios, 1-10.

Giorgetti, Romero, \& Vera. (2014). Estudio de los modelos de evaluación de la calidad existentes para la conceptualización de un modelo adecuado para Instituciones de Educación
Superior. ISBN: 978-84-7666210-6 - Artículo 1466, 1-20.

González B. (2014). Diseño de un modelo de gestion generico para unidades académicas. Tesis de Maestria, Pontificia Universidad Catolica del Ecuador, Ambato.

Guananga, L. (2018). Situacion y gestion del emprendimiento. ISBN: 978-9942-30-580-0, 9100.

Jaime Carriel, E. (2017). El emprendimiento en Ecuador, Vision y perpectivas. Universidad ECOTEC ISBN 978-9942-960-28-3, 7-90.

Lasio, V. et al. (2017). Espae - Espol $2018 . \quad$ Global Entrepreneurship Monitor Ecuador, 4-80. doi:ISSN No. 13903047

Maldonado, P. (2019). "A emprender con calidad". https://www.elcomercio.com/ blogs/a-innovar/emprendercalidad-pedro-maldonadoopinion.html

Rodríguez, A. y Sandoval, L. (2017). Modelo Malcolm Baldrige para la gestión de calidad total. SCIELO, 1-15.

Sáinz, E. (2018). Aplicación del modelo EFQM para la mejora continua de la calidad. Tesis doctoral, Universidad Complutense de Madrid, Madrid.

Santander, U. I. (2011). UniversidadEmpresa-Estado. 
Universidad-Empresa-

Estado:

http://planmaestroinv.udistrita I.edu.co/documentos/PMIClUD/unidadtransfer/UIS/UNIV ERSIDAD\%20\%20EMPRESA\%20ESTADO(Vic\%20Acad\%C3\% A9mica).pdf

Serna, E. (2019). Desarrollo e Innovación en Ingeniería. Instituto Antioqueño de Investigación.

doi:http://doi.org/10.5281/zen odo.3387679

Zamora, C. (2017). "Importancia del emprendimiento en la economía: caso Ecuador". https://www.revistaespacios.c om/a18v39n07/a18v39n07p1 5.pdf 